\title{
The Radio Remnant of Supernova 1987A - A Broader View
}

\author{
G. Zanardo ${ }^{1}$, L. Staveley-Smith ${ }^{1,2}$ C. -Y. Ng ${ }^{3}$, R. Indebetouw ${ }^{4}$, \\ M. Matsuura ${ }^{5,6}$, B. M. Gaensler ${ }^{7,2}$ and A. K. Tzioumis ${ }^{8}$ \\ ${ }^{1}$ International Centre for Radio Astronomy Research (ICRAR), \\ M468, The University of Western Australia, Crawley, WA 6009, Australia. \\ email: giovanna.zanardo@gmail.org \\ ${ }^{2}$ Australian Research Council Centre of Excellence for All-sky Astrophysics (CAASTRO) \\ ${ }^{3}$ Department of Physics, The University of Hong Kong, Pokfulam Road, Hong Kong \\ ${ }^{4}$ Department of Astronomy, University of Virginia, P.O. Box 400325, \\ Charlottesville, VA 22904-4325, USA \\ ${ }^{5}$ School of Physics and Astronomy, Cardiff University, Queens Buildings, The Parade, \\ Cardiff, CF24 3AA, UK \\ ${ }^{6}$ Department of Physics and Astronomy, University College London, Gower Street, \\ London, WC1E 6BT, UK \\ ${ }^{7}$ CSIRO Astronomy and Space Science, Australia Telescope National Facility, \\ PO Box 76, Epping, NSW 1710, Australia \\ ${ }^{8}$ Dunlap Institute for Astronomy \& Astrophysics, University of Toronto, \\ Toronto, ON M5S 3H4, Canada
}

\begin{abstract}
Supernova remnants (SNRs) are powerful particle accelerators. As a supernova (SN) blast wave propagates through the circumstellar medium (CSM), electrons and protons scatter across the shock and gain energy by entrapment in the magnetic field. The accelerated particles generate further magnetic field fluctuations and local amplification, leading to cosmic ray production. The wealth of data from Supernova 1987A is providing a template of the SN-CSM interaction, and an important guide to the radio detection and identification of core-collapse $\mathrm{SNe}$ based on their spectral properties. Thirty years after the explosion, radio observations of SNR 1987A span from $70 \mathrm{MHz}$ to $700 \mathrm{GHz}$. We review extensive observing campaigns with the Australia Telescope Compact Array (ATCA) and the Atacama Large Millimeter/submillimeter Array (ALMA), and follow-ups with other radio telescopes. Observations across the radio spectrum indicate rapid changes in the remnant morphology, while current ATCA and ALMA observations show that the SNR has entered a new evolutionary phase.
\end{abstract}

Keywords. circumstellar matter, ISM: supernova remnants, radio continuum: general, supernovae: individual (SN 1987A), acceleration of particles, radiation mechanisms: nonthermal

\section{Background}

Radio supernovae are most consistently identified with core-collapse supernovae (CC$\mathrm{SNe}$ ) and their transition into supernova remnants (SNRs). The radio emission results from the collision of the supernova $(\mathrm{SN})$ shock and the progenitor's circumstellar medium (CSM). Many radio SNe can be associated with HII regions or regions of active star formation. Given the high ambient pressure of the surrounding HII region, radio SNe and their remnants are primarily identified by dense CSM environments. The SN shock wave modifies the CSM as it passes through it, both by compression and shock heating. The 
forward SN shock, i.e. the outer blast wave, and the reverse shock, which causes deceleration and heating of the inner ejecta, accelerate the shocked material, with some fractions of electrons and protons reaching cosmic ray energies. The acceleration mechanism usually associated with the shock propagation is diffusive shock acceleration (DSA), also known as the first-order Fermi mechanism (Drury 1983). According to DSA theory, energetic particles at shock fronts undergo spatial diffusion in a uniform magnetic field. If the magnetic field is not uniform, as in the case of young SNRs, the charged particles can get partially trapped in the magnetic field structure, while still gaining energy by scattering between the upstream and downstream regions of the forward shock (Kirk et al. 1996). This leads to a sub-diffusive particle transfer (sub-DSA), since the particle density at the shock front is lower than it is far downstream. In either case, as energetic electrons gyrate in the local magnetic field, they emit synchrotron emission. The synchrotron emission observed in SNRs can be well described by a power law $S_{\nu} \propto \nu^{\alpha}$, with $S_{\nu}$ the flux density measured at the observing frequency $\nu$. Simplified synchrotron physics tells us that emission at the frequency $\nu$ is primarily by electrons with energy $E \propto 15 \sqrt{\nu / B} \mathrm{GeV}$, with $\nu$ expressed in $\mathrm{GHz}$ and the magnetic field strength, $B$, expressed in $\mu \mathrm{G}$ (Reynolds 2011).

SN 1987A in the Large Magellanic Cloud, identified as Type IIP (Leibundgut \& Suntzeff 2003) with progenitor of mass $\sim 20 M_{\odot}$ (Smartt 2009), is the only nearby CCSN observed with a telescope since its early stages. Given its proximity, SN 1987A has allowed unique studies of the radio evolution of CCSNe and of the SN-CSM interaction. The complex CSM distribution in the remnant is believed to have originated from a red supergiant (RSG) which has evolved into a blue supergiant (BSG) about 20,000 years before the explosion (Crotts \& Heathcote 2000). In its transition from supernova to SNR, SN 1987A has continued to brighten for the past three decades, thus making possible to monitor the rapid changes of the radio emission with a number of telescopes over an increasingly broad frequency range.

\section{Evolution of the SN-CSM interaction}

The interaction of the SN shock wave with the CSM in the equatorial plane (equatorial ring, ER) is the most notable feature of the radio remnant of SN 1987A. The ER defines the waist of the 'hourglass' environment created by the progenitor star prior to explosion. As the SN propagates outwards and impacts the denser CSM in the ER, the shocks expand above and below the ER plane (see Figure 1) and interact with high-latitude material (Potter et al. 2014), confined within the inner hourglass structure.

\subsection{Light curves}

The radio emission from SN 1987A was first detected two days after the arrival of the neutrinos with the Molonglo Observatory Synthesis Telescope (MOST) at $843 \mathrm{MHz}, 1.4$, 2.3, and $8.4 \mathrm{GHz}$ (Turtle et al. 1987). The peak flux density was reached at $\sim 130 \mathrm{mJy}$ at $843 \mathrm{MHz}$ four days after the explosion (Turtle et al. 1987), followed by a power-law decay of the emission, which became undetectable from September 1987 (Ball et al. 1995). It is understood that this radio outburst was due to the faster BSG wind, which produced a short-lived radio emission when hit by the SN shock (Chevalier \& Fransson 1987). The radio emission re-appeared above noise $\sim 1200$ days after the explosion (StaveleySmith et al. 1992), and has been monitored with the Australia Telescope Compact Array (ATCA) since then. Gaensler et al. (1997) calculated that, between the SN event and the second radio turn-on in mid-1990, the radio emitting regions expanded at an average velocity of $\sim 35,000 \mathrm{~km} \mathrm{~s}^{-1}$, which is possible in a very low-density inner CSM such as 


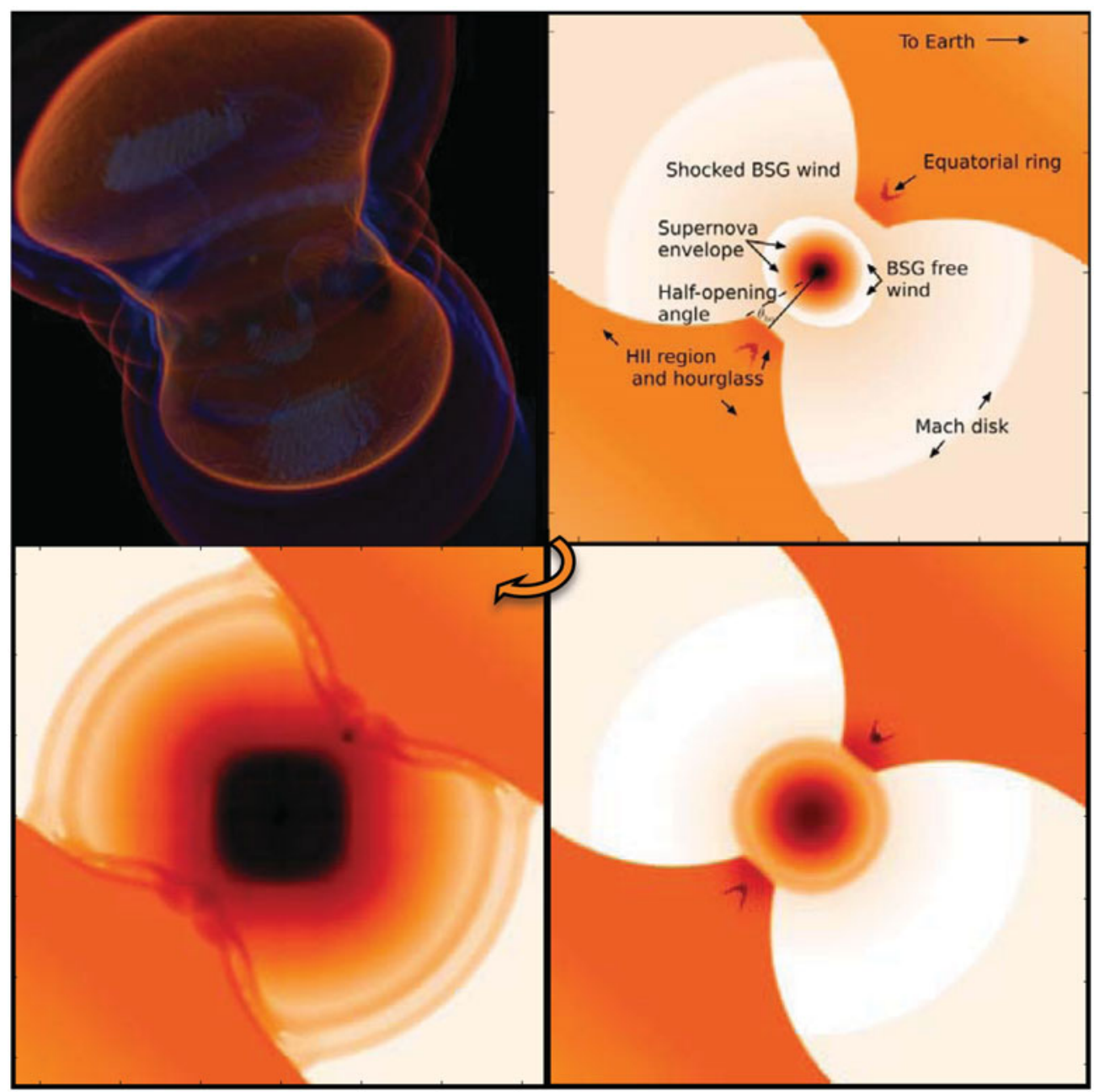

Figure 1. Stages of the shock expansion from HD simulations by Potter et al. (2014) (see their Figures 1, 6, and 7), as modelled around 1000 days (top right), at day $\sim 2000$ (bottom right), and at day $\sim 8000$ (bottom left) since the SN. By day $\sim 8000$, the forward shock has overtaken the equatorial ring (ER). The colour scheme varies from white to dark brown, indicating the increasing particle number density in $\log _{10}$ scale $\left(\sim 5-11 \times 10^{7} \mathrm{~m}^{-3}\right.$, see Potter et al. 2014$)$. A $3 \mathrm{D}$ view of both the forward and reverse shocks expanding above and below the ER, is shown on the top left image, which is a rendering of the volumetric models developed by Potter et al. (2014) (see their Figure 9) at day 8000. Source: Potter et al. (2014).

that surrounding Type Ib/Ic SNe. The expansion velocity sharply decreased to $v_{s} \lesssim 7000$ $\mathrm{km} \mathrm{s}^{-1}$ from day $\sim 1200$ (Gaensler et al. 1997), when the radio emission started to increase (Ball et al. 2001). Since day $\sim 5000$ the radio emission has been rising at an exponential rate (Zanardo et al. 2010, Figure 2). A new phase of the SN-CSM interaction is observed at all frequencies after day $\sim 8000$ (Figure 2; Zanardo 2014), when the shock has likely become engulfed in the densest CSM in the ER, while still expanding above and below the ring.

\subsection{Morphology}

The morphology of the non-thermal radiation emitted by relativistic electrons accelerated in the remnant has been monitored with the Australia Telescope Compact Array (ATCA) 

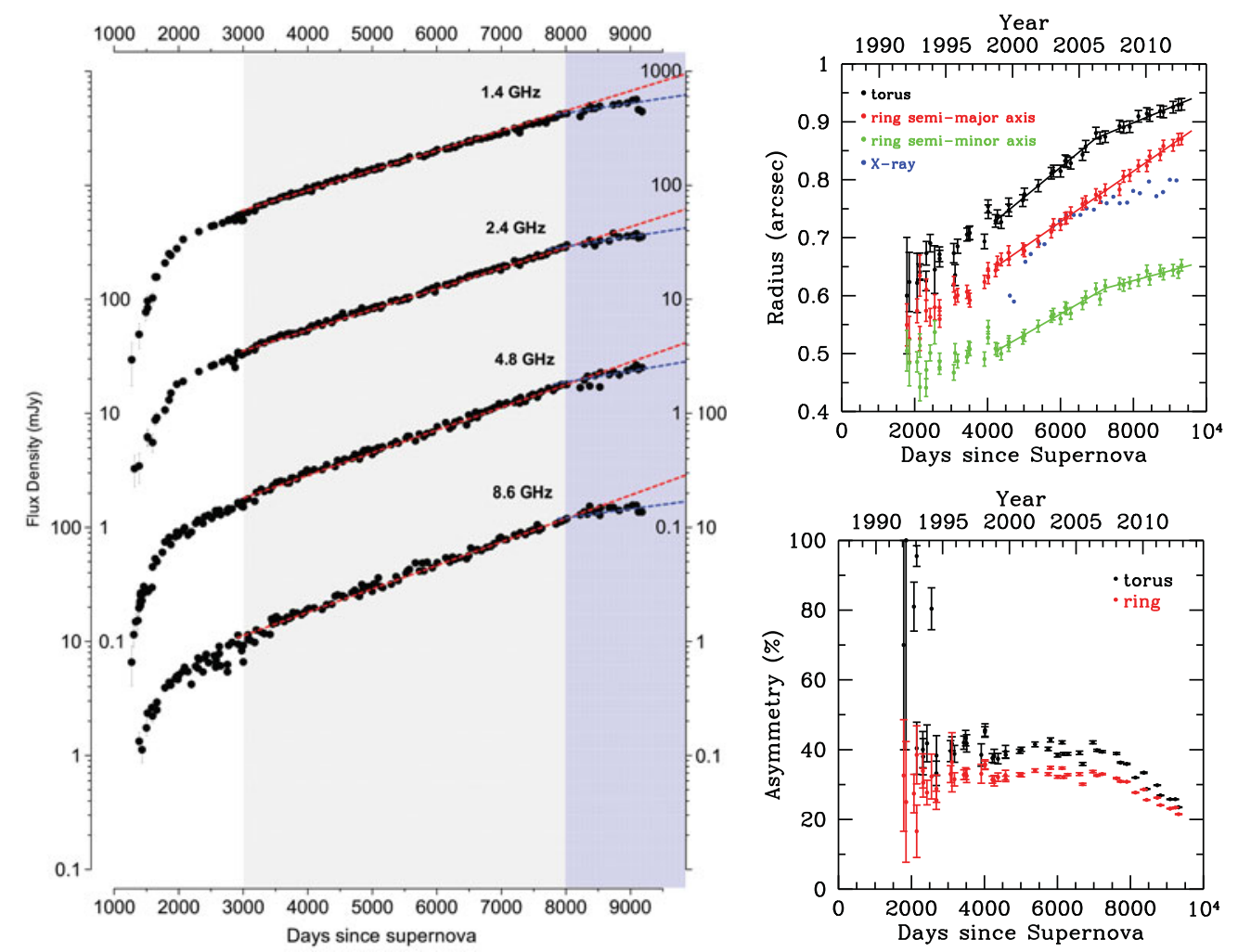

Figure 2. Left - Flux densities for SNR 1987A. Plots include data from observations at 1.4, 2.4, 4.8, and $8.6 \mathrm{GHz}$ from August 1990 to June 2012 and are stacked with an offset of $0.1 \mathrm{mJy}$ (Zanardo et al. 2010; Zanardo 2014). The red dashed lines are the exponential fits of data from November 2000 to February 2009, as derived by Zanardo et al. (2010). The blue dashed lines are 1500-day fits of data from February 2009. The colour bands are included to highlight different rates of the flux density increase. Top Right - Remnant radius resultant from images at $9 \mathrm{GHz}$, from August 1992 to March 2013, as derived by Ng et al. (2013) via fitting the SNR with a torus (black) and an elliptical ring (red: semi-major axis; green: semi-minor axis). The radius of the radio remnant is compared with that derived from X-ray images (blue, from Helder et al. 2013). The solid lines indicate the best-fit expansion. Bottom Right - Evolution of the remnant asymmetry at $9 \mathrm{GHz}$ as derived from the torus (black) and ring (red) models ( $\mathrm{Ng}$ et al. 2013). Source: Zanardo (2014), Ng et al. (2013).

since 1992 using images at $9 \mathrm{GHz}$ (Staveley-Smith et al. 1992), with a spatial resolution of 0.5 (Gaensler et al. 1997; Ng et al. 2008). These images have provided insight into the trademarks of the radio emission (see Figure 3; $\mathrm{Ng}$ et al. 2013), such as: (a) the steady brightening; $(b)$ the ring-like structure evolved from a two-lobe morphology; $(c)$ the east-west asymmetry, with brightness peak on the eastern lobe. As discussed by $\mathrm{Ng}$ et al. (2013) (left plots in Figure 2), by day 8000 the faster eastbound shocks (Zanardo et al. 2013) have possibly overtaken the ER. This would have resulted in a reduction of the radio emission from the eastern rim and, thus, impacted the overall brightness asymmetry (see Figure 2). High-resolution images obtained at $44 \mathrm{GHz}$ with the ATCA, and at $1.4 \mathrm{GHz}$ via very-long baseline interferometry (VLBI) with the Australian Large Baseline Array (LBA; see Figure 4; Zanardo 2014), have shown small-scale structures more prominent on the eastern lobe, which are likely due to the shock wave interacting with discrete clumps near the inner surface of the SN shell (see Ng et al. 2011). 


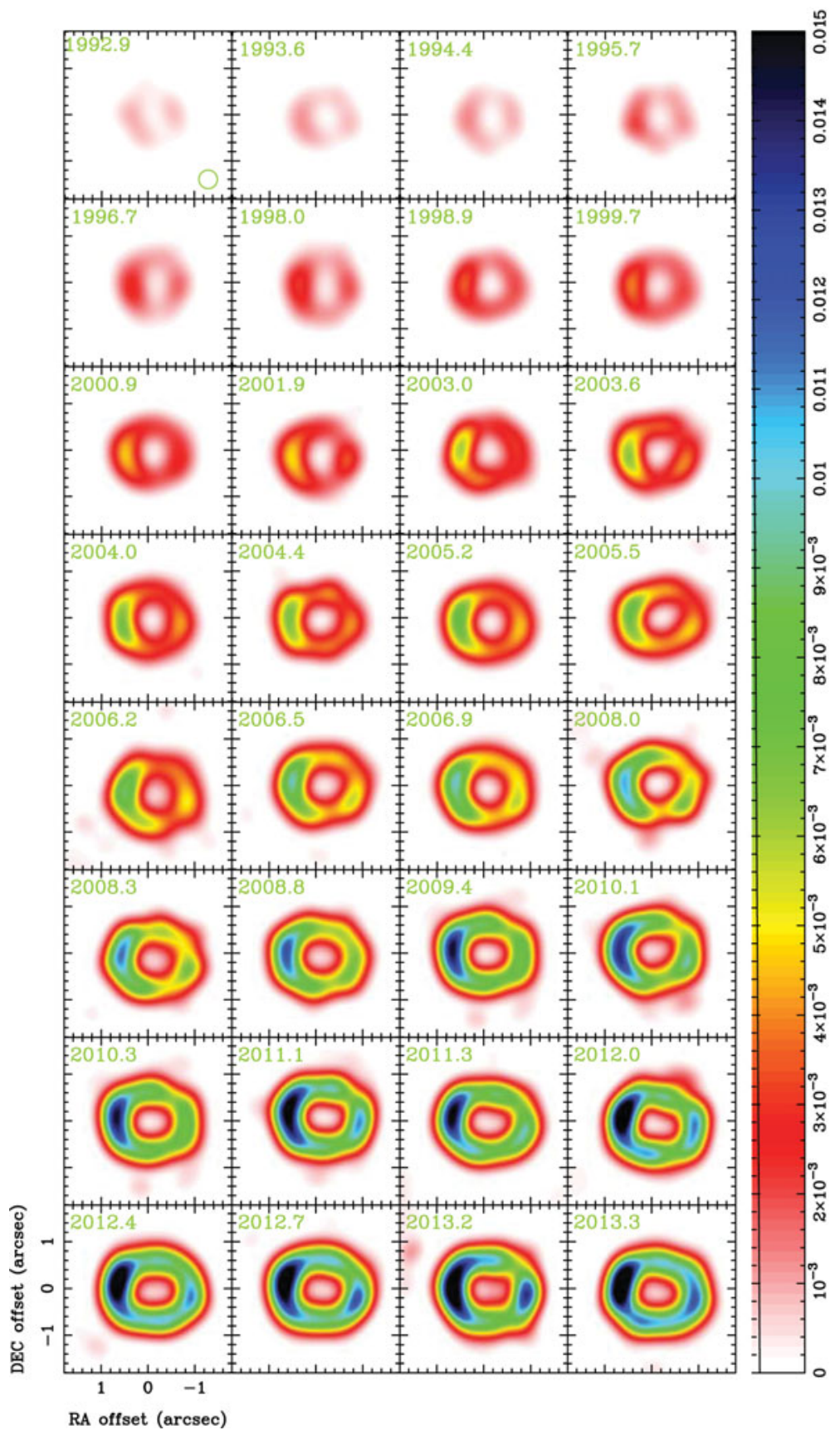

Figure 3. $9 \mathrm{GHz}$ images of SNR 1987A from 1992 to 2013, adapted from Ng et al. (2013) (see Figure 1 therein). Images include data from Gaensler et al. (1997), Ng et al. (2008), and $\mathrm{Ng}$ et al. (2013). Colour-bar units are Jy beam ${ }^{-1}$. Source: Ng et al. (2013), Zanardo (2014).

\subsection{Asymmetry}

The remnant asymmetry has been confirmed in all observations from radio to sub-mm (see Figure 5) and discussed for observations at contemporaneous epochs. The east-west asymmetry ratio is $1.4-1.5$ at 18 and $44 \mathrm{GHz}$ (Zanardo et al. 2013), and it shows a gradual 


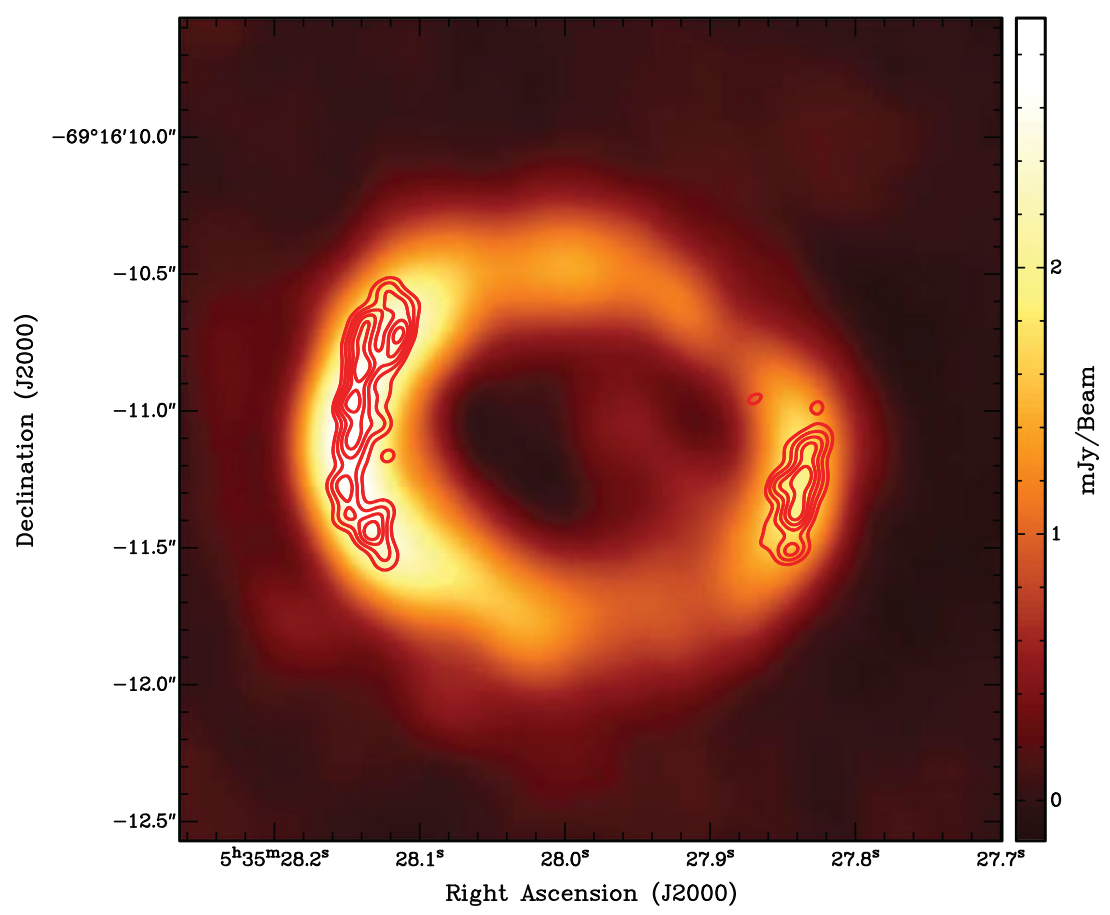

Figure 4. Contours of the 2010 VLBI image of SN 1987A at $1.4 \mathrm{GHz}$ are overlaid with the $44 \mathrm{GHz}$ image derived from 2011 observations (Zanardo et al. 2013). The $44 \mathrm{GHz}$ image has a resolution of $0{ }^{\prime \prime} 2$, while the VLBI image has a restoring beam of $61 \times 95$ mas. The VLBI observations have been instrumental to align images prior to the derivation of spectral index maps (see Zanardo et al. 2013; Zanardo et al. 2014). Credit: Zanardo (2014).

decrease in images from 44 to $345 \mathrm{GHz}$ (Zanardo et al. 2014), where there are hints of a reversed trend. As the synchrotron lifetime becomes shorter at higher frequencies, there would be regions in the eastern side of the remnant where electrons might be unable to cross the emission sites within their radiative life-time. In this scenario, the synchrotron emission would require the presence of relatively fresh injected and/or re-accelerated electrons to match the emission distribution at lower frequencies. Zanardo et al. (2014) estimated that, at frequencies higher than $213 \mathrm{GHz}$, the electrons might be unable to cross the emission regions on the eastern lobe.

Further, the asymmetry in the shock expansion velocities, with significantly faster eastbound shocks (Zanardo et al. 2013), has been related to an asymmetric explosion (Zanardo et al. 2013; Zanardo et al. 2014). The possibility of an asymmetric initial explosion was suggested by Chevalier \& Soker (1989), while Podsiadlowski, Morris \& Ivanova (2007) proposed a binary merger to reproduce the BSG progenitor and the rings formed from the BSG-RSG wind interaction. Although marked asymmetries have also been seen in the inner ejecta, (Kjær et al. 2010; Larsson et al. 2013), the recent detection of ${ }^{44} \mathrm{Ti}$ emission with the Nuclear Spectroscopic Telescope Array (NuSTAR) provides direct evidence of large-scale asymmetry in the explosion (Boggs et al. 2015).

\subsection{Spectral index}

A progressive hardening of the radio spectra obtained from ATCA multi-frequency flux monitoring has been observed since day $\sim 2500$ (Zanardo et al. 2010), and up to day $\sim 8000$. The results have pointed to sub-DSA particle transport (Kirk et al. 1996), which leads to increasingly efficient particle acceleration and cosmic ray production. On a local 


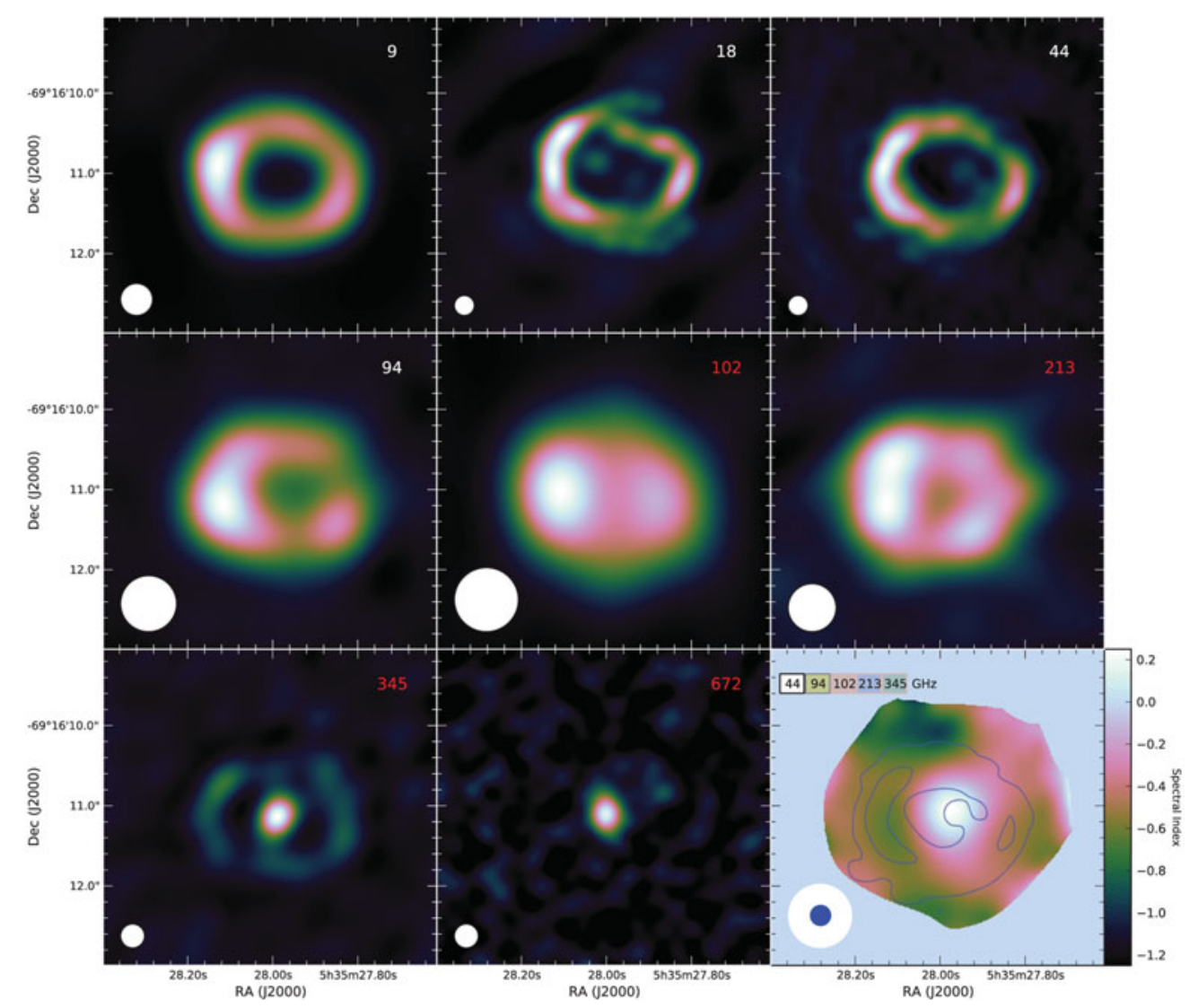

Figure 5. Mosaic of radio images of the remnant of SN 1987A at day 9280. Each image frequency is indicated on the top right corner, in $\mathrm{GHz}$. Images below $100 \mathrm{GHz}$ are derived from ATCA observations (Lakićević et al. 2012a; Ng et al. 2013; Zanardo et al. 2013), images above 100 $\mathrm{GHz}$ are from ALMA data (Zanardo et al. 2014). All images have been super-resolved with a circular beam (lower left corner). The bottom right image is the spectral index map obtained by combining images at five frequencies, i.e. 44, 94, 102, 213, and $345 \mathrm{GHz}$ (see Zanardo et al. 2014). The spectral image is overlaid with contours (in blue) of the $15 \%$ and $60 \%$ emission levels at $44 \mathrm{GHz}$ (Zanardo et al. 2013). Credit: Zanardo (2014).

scale, high-resolution spectral maps have consistently shown steeper synchrotron spectral indices and, thus, lower compression ratios, mainly localised on the eastern lobe of the remnant (see Figure 5; Zanardo et al. 2013; Zanardo et al. 2014). A softer particle spectrum on the eastern regions and the higher Mach numbers measured for the eastbound shocks (Zanardo et al. 2013), are likely to create the conditions for magnetic field amplification (Bell \& Lucek 2001) with efficient cosmic ray production. Local magnetic field amplification is compatible with significant synchrotron losses at higher frequencies, which can explain the decrease of the east-west asymmetry ratio of the emission in the transition from radio to sub-millimetre wavelenghts (Zanardo et al. 2014).

The spectral energy distribution (SED) from 1.4 to $3000 \mathrm{GHz}$ (see Figure 6) for 2011-2012 data yields a 'global' synchrotron spectral index $\alpha=-0.73$, which is confirmed in observations at low frequencies $(72-230 \mathrm{MHz})$ carried out with the Murchison Widefield Array (MWA) in late 2013 (Callingham et al. 2016). The absence of a spectral turnover down to $72 \mathrm{MHz}$, places an upper limit of $n_{e}=110 \mathrm{~cm}^{-3}$ to the electron density of the medium in which the SN radio emission propagates (Callingham et al. 2016). 


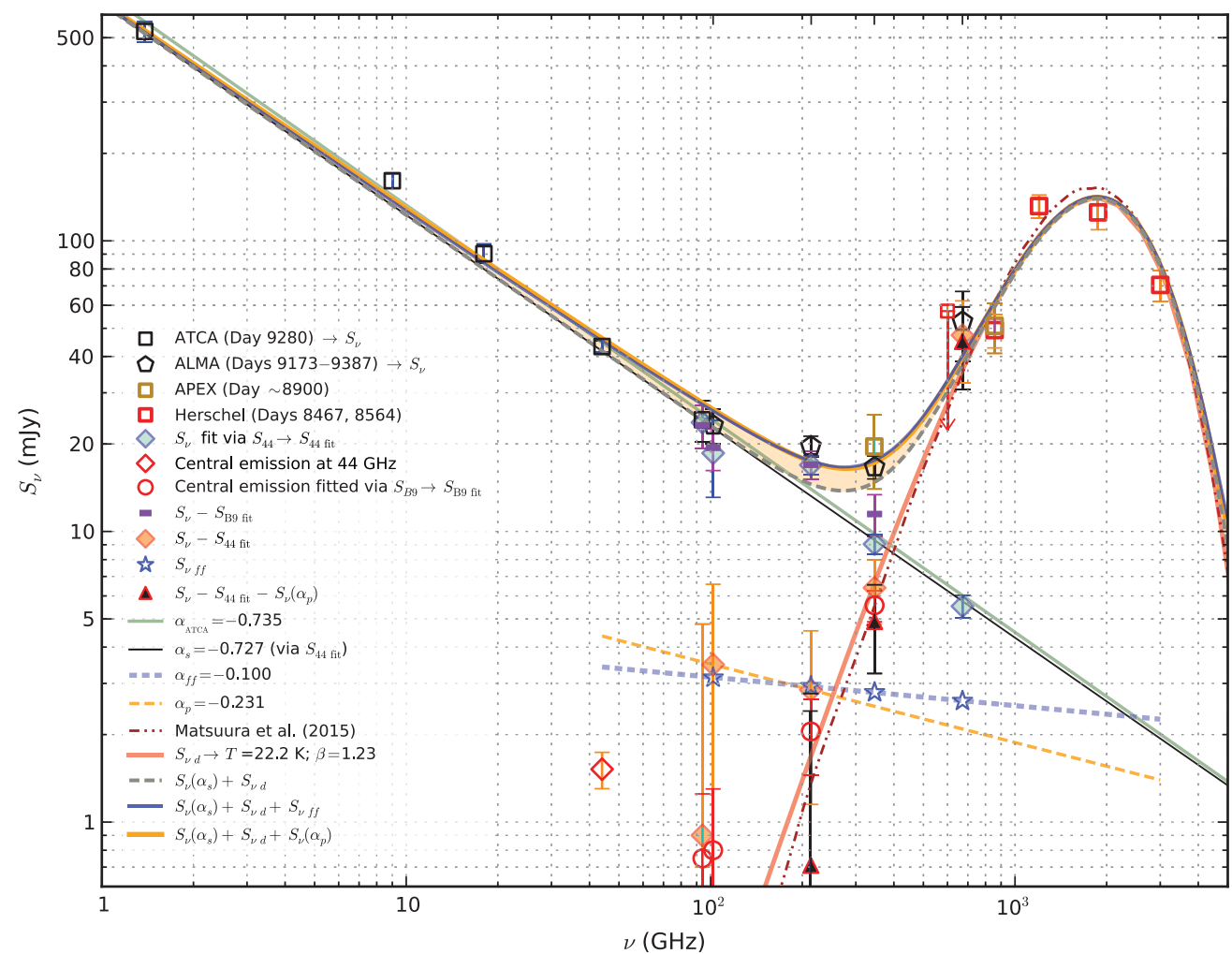

Figure 6. SED of SNR 1987A from radio to FIR, with data from: ATCA at $1.4 \mathrm{GHz}$ (Zanardo 2014), $9 \mathrm{GHz}$ (Ng et al. 2013), 18 and $44 \mathrm{GHz}$ (Zanardo et al. 2013), and $94 \mathrm{GHz}$ (Lakićević et al. 2012a); ALMA (Zanardo et al. 2014); the Atacama Pathfinder EXperiment (APEX) at 345 and $857 \mathrm{GHz}$ (Lakićević et al. 2012b); the Herschel Space Observatory at $600-3000 \mathrm{GHz}$ (Matsuura et al. 2011). The brown dash-dot-dotted curve is the amorphous carbon dust fit for ALMA data and Herschel observations carried out in 2012 (Matsuura et al. 2015). To match the average epoch of ALMA observations, ATCA data are scaled to day 9280, via exponential fitting parameters derived for ATCA flux densities from day 8000, as measured at 1.4, 8.6 and $9 \mathrm{GHz}$ (Zanardo 2014) The hollow red diamond indicates the central emission measured at 44 $\mathrm{GHz}$ as reported by Zanardo et al. (2013) scaled to day 9280. The difference between possible spectral fits is highlighted in light orange. Credit: Zanardo et al. (2014).

\section{Compact remnant}

The search for a compact object in the inner regions of SNR 1987A has been undertaken on multiple fronts. It has been shown that flat spectral synchrotron indices can be associated either with accretion onto a black hole or with a pulsar wind nebula (PWN; Gaensler \& Slane 2006). Spectral index maps of the remnant derived at different frequencies and epochs have consistently featured a flat central component (Lakicević et al. 2012a; Potter et al. 2009; Zanardo et al. 2013; Zanardo et al. 2014).

The discovery with ALMA (Indebetouw et al. 2014; Kamenetzky et al. 2013) and Herschel (Matsuura et al. 2011, 2015) of $\sim 0.8 M_{\odot}$ of newly-forming dust grains in the SNR interior, complicates further the detection of a PWN expected to be expanding into the inner ejecta. The dust sits inside the ER and overlaps with the ejecta as seen with HST (Figure 7). Combined ALMA and ATCA observations have allowed one to separate the thermal and non-thermal components of the SNR emission (Indebetouw et al. 2014; Zanardo et al. 2014). After subtraction of the dust emission and the synchrotron 


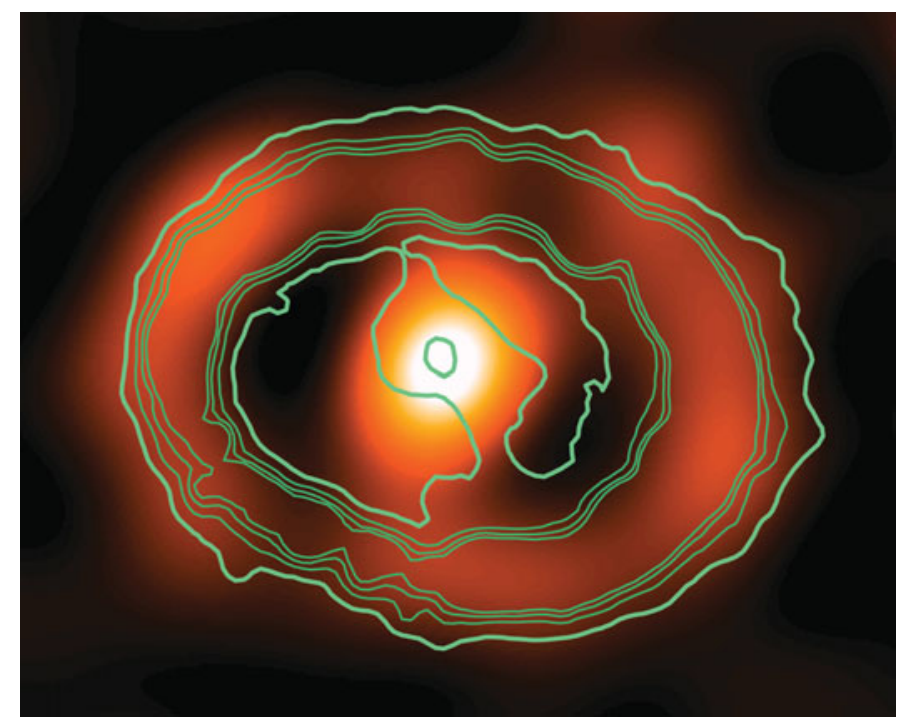

Figure 7. An outline of the equatorial ring and inner debris, as seen with HST (green/blue contours), on top of ALMA observations of the remnant at $345 \mathrm{GHz}$ (Zanardo et al. 2014) (red/orange). The dust in the central region is rendered. (Hubble data: NASA/STScI/CfA/P. Challis and R. Kirshner.)

emission from the outer shock, the residual emission of $\sim 3$ mJy (see Figure 6 ) in the central region could correspond to the synchrotron emission from an embedded pulsar, possibly located at $\sim 50$ mas from the SN site. This PWN position would match the centre of the inner emission detected at $44 \mathrm{GHz}$ (Figure 4), and would imply a natal kick of $\sim 400 \mathrm{~km} \mathrm{~s}^{-1}$, for a possible pulsar period of $\sim 150 \mathrm{~ms}$ (Zanardo et al. 2014). An asymmetric explosion of the progenitor could have led to a high-velocity neutron star (Janka 2012) and, thus, to the formation of a PWN offset from the SN site.

After unsuccessful searches for the pulsar signal with the Parkes telescope (Manchester $1988,2007)$, the preliminary analysis of data from an extended pulsar search campaign in 2013 has tentatively identified several candidates with period $30 \lesssim P \lesssim 300 \mathrm{~ms}$ (Zanardo 2014). However, there is yet no convincing detection and the search continues.

\section{References}

Ball, L., Campbell-Wilson, D., Crawford, D. F., \& Turtle, A. J. 1995, ApJ., 453, 864

Ball, L., Crawford, D. F., Hunstead, R. W., Klamer, I., \& McIntyre, V. J. 2001, ApJ, 549, 599

Bell, A. R. \& Lucek, S. G. 2001, MNRAS, 321, 433

Bell, A. R. 2004, MNRAS, 353, 558

Boggs et al. 2015, Science, 348, 670

Callingham, J. R., Gaensler, B. M., Zanardo, G. et al. 2016, MNRAS, 462, 290

Chevalier, R. A. \& Fransson, C. 1987, Nature, 328, 44

Chevalier, R. A. \& Soker, N. 1989, ApJ.. 341, 867

Crotts, A. P. S. \& Heathcote, R. S. 2000, ApJ, 528, 426

Drury, L. Oc. 1983, Rep. Prog. Phys., 46, 973

Gaensler, B. M., Manchester, R. N., Staveley-Smith, L. et al. 1997, ApJ, 479, 845

Gaensler, B. M. 1998, ApJ, 493, 781

Gaensler, B. M. \& Slane, P. O. 2006, ARAA, 44, 17

Helder, E. A., Broos, P. S., Dewey, D. et al. 2013, ApJ, 764, 11

Indebetouw, R., Matsuura, M., Dwek, E., et al. 2014, ApJ., 782, L2 
Janka, H.-T. 2012, Annual Review of Nuclear and Particle Science, 62, 407

Kamenetzky, J., McCray, R., Indebetouw, R., et al. 2013, ApJ., 773, L34

Kjær K., Leibundgut, B., Fransson, C. et al. 2010, A\&A, 517, A51

Kirk, J. G., Duffy P. \& Gallant, Y. A. 1996, A\&SA, 314, 1010

Lakićević, M., Zanardo, G., van Loon, J. Th., et al. 2012a, A\&A, 541, L2

Lakićević, M., van Loon, J. Th., Stanke, T. et al. 2012b, A\& A, 541, L1

Larsson, J., Fransson, C., Kjær, K., et al. 2013, ApJ, 768, 89

Leibundgut, B. \& Suntzeff, N. B. 2003, Supernovae and Gamma-Ray Bursters, edited by K.

Weiler, Lecture Notes in Physics, vol. 598, p.77-90

Manchester, R. N. 1988, PASA, 7, 548-549.

Manchester, R. N. 2007, AIP Conf. Proc., 937, 134-143

Matsuura, M., Dwek, E., Meixner, M., et al. 2011, Science, 333, 1258

Matsuura, M., Dwek, E., Barlow, M. J. et al. 2015, ApJ., 800, 50

Matsuura, M., Indebetouw, R., Woosley, S. et al. 2017, MNRAS, in press, arXiv:1704.02324

Ng, C.-Y., Gaensler, B. M., Staveley-Smith, L. et al. 2008, ApJ., 684, 481

Ng, C.-Y., Potter, T. M., Staveley-Smith, L. et al. 2011, ApJ (Letters), 728, L15

Ng, C.-Y., Zanardo, G., Potter, T. M., et al. 2013, ApJ., 777,131

Podsiadlowski, Ph., Morris, T. S., \& Ivanova, N. 2007, AIP Conf. Proc., 937, 125-133

Potter, T. M., Staveley-Smith, L., Ng, C.-Y. et al. 2009, ApJ, 705, 261

Potter, T. M., Staveley-Smith, L., Reville, B., et al. 2014, ApJ., 794, 174

Reynolds, S. P. 2011, ApE्SSS, 336, 257

Smartt, S. J. 2009, ARAA, 47, 63

Staveley-Smith, L. et al. 1992, Nature, 355, 147

Turtle, A. J., Campbell-Wilson, D., Bunton, J. D. et al. 1987, Nature, 327, 38

Turtle, A. J., Campbell-Wilson, D., Manchester, R. N. et al. 1990, IAU Circ. 5086, 2

Zanardo, G., Staveley-Smith, L., Ball, Lewis et al. 2010 ApJ, 710, 1515

Zanardo, G., Staveley-Smith, L., Ng, C.-Y. et al. 2013, ApJ, 767, 98

Zanardo, G., Staveley-Smith, L., Indebetouw, R., et al. 2014, ApJ., 796, 82

Zanardo, G. 2014, Radio Evolution of the Remnant of Supernova 1987A, PhD Thesis, University of Western Australia. 